\title{
SIAT-WEXv2: A Wearable Exoskeleton for Reducing Lumbar Load during Lifting Tasks
}

\author{
Xinyu Ji, ${ }^{1,2,3,4}$ Dashuai Wang, ${ }^{1,2,4}$ Pengfei Li, ${ }^{1,2,4}$ Liangsheng Zheng, ${ }^{1,2,4}$ Jianquan Sun, ${ }^{1,2,4}$ \\ and Xinyu $W u$ iD ${ }^{1,2,4}$ \\ ${ }^{1}$ Guangdong Provincial Key Lab of Robotics and Intelligent System, Shenzhen Institutes of Advanced Technology, \\ Chinese Academy of Sciences, Shenzhen, China \\ ${ }^{2}$ CAS Key Laboratory of Human-Machine Intelligence-Synergy Systems, Shenzhen Institutes of Advanced Technology, \\ Shenzhen, China \\ ${ }^{3}$ Wuhan University of Technology, Wuhan, China \\ ${ }^{4}$ Guangdong-Hong Kong-Macao Joint Laboratory of Human-Machine Intelligence-Synergy Systems, Shenzhen, China
}

Correspondence should be addressed to Xinyu Wu; xy.wu@siat.ac.cn

Received 7 August 2020; Revised 6 September 2020; Accepted 17 September 2020; Published 28 November 2020

Academic Editor: Zhile Yang

Copyright $(2020$ Xinyu Ji et al. This is an open access article distributed under the Creative Commons Attribution License, which permits unrestricted use, distribution, and reproduction in any medium, provided the original work is properly cited.

Lumbar Exoskeleton, as an important instance of wearable exoskeleton, has broad application prospects in logistics, construction, and other industries. Specifically, in the working scenarios that require long-term and repeated bending and rising movements, active lumbar exoskeleton (ALE) can provide effective protection and flexible assistance to wear's waist muscles and bones, which will significantly reduce the risk of lumbar muscle strain. How to improve the human-machine coupling and enhance the assistance performance are the main challenges for ALE's development. Based on the biomechanical analysis of the movement of lifting heavy objects from bottom up, this paper proposes a lightweight but powerful ALE, named as SIAT-WEXv2, which can output maximum assistive force of $28 \mathrm{~N}$. Additionally, we use robust fuzzy adaptive algorithm to improve SIAT-WEXv2's antidisturbance ability, so that it can provide continuous and supple assistance for wearer. Electromyography (EMG) signals of the lumbar erector spinae (LES) from ten subjects in two experimental cases (with or without SIAT-WEXv2) were collected to evaluate the effectiveness of our new ALE. The experimental results indicate that the reduction of iEMG signal at LES decreased monotonically from $60 \% \pm 5.5 \%$ to $40.5 \% \pm 6.5 \%$ as the weight of lifting load increased from 0 to $25 \mathrm{~kg}$.

\section{Introduction}

Musculoskeletal diseases are the most common diseases in many industrial countries, for example, China, India, and America. They have a major impact on the quality of workers' life and the loss of company's productivity. The proportion of workers with lumbar problems in the logistics, construction, and medical rehabilitation industries is $84 \%$, $75 \%, 75 \%$, and $67 \%$, respectively [1]. This problem has become a social hazard. To solve this problem, many solutions have been proposed. Among them, the research of the lumbar exoskeleton is worthy of attention [2].

According to statistics, when wearers were using ALE, the lumbar spine muscle activity can be reduced by up to
$30 \%$ [3]. In order to improve its force-assisted performance, many universities and research institutions are developing lighter and more powerful lumbar exoskeletons. Due to the different driving components of the exoskeleton, it can be divided into two types: passive and active [2].

Varieties of passive exoskeletons have been developed, for instance, PLAD [4-6], WMRD [7], BNDR [8], VT/Lower [9], SPEXOR [10], Laevo [11], and BackX [12]. PLAD uses elastic bands to reduce the muscle load of the vertical spine when lifting. It reduces spinal muscle activity (including the average amount of muscle activity in the chest and lumbar) during flexion (37\%), squat (38\%), and freestyle (37\%) weightlifting. WMRD uses helical springs. With the device, back muscles demonstrated a 54\% reduction in muscle 
activity. BNDR uses torsion springs to reduce the lumbar load and does not increase the load on the hips, knees, and ankles. When wearing the device in a bent posture, the compression and shear forces at the L5-S1 level were reduced by $13 \%$ and $12 \%$, respectively. The internal load of the leg joints is reduced by $10 \%$ to $31 \%$. VT/Lower uses carbon fiber as an energy storage material, and its energy feedback is much higher than that of a gas spring. Using this device, the peak and average activity of the back muscles during symmetrical weightlifting were reduced by $31.5 \%$ and $29.3 \%$, respectively. SPEXOR uses both coil springs and flexible beams to provide power. After wearing the exoskeleton, the metabolic cost was reduced by $18 \%$, and the muscle activity was reduced by $16 \%$. Both Laevo (weight: $2.8 \mathrm{~kg}$ ) and BackX (weight: $3.3 \mathrm{~kg}$ ) use gas springs to provide lumbar support to users. These passive exoskeletons are not only lightweight but also provide force to reduce lumbar muscle fatigue.

However, these passive exoskeletons cannot provide enough power, so the reduction in muscle activity is limited. To solve this problem, researchers began to develop active lumbar exoskeletons. For example, H-WEX v1 [13], H-WEX v2 [14], lower back exoskeleton [15], MRLift [16], muscle suit [17], ATOUN MODEL Y [18], ROBO-MATE [19], and SIAT-WEXv1 [20]. H-WEXv1 uses only one actuator to assist the worker for lifting tasks. And, activities of major muscles related to lumbar motions were reduced by $10 \%$ to $30 \%$. H-WEXv2 (weight: $5.5 \mathrm{~kg}$ ) utilizes SEAbased wire-driven mechanism to achieve system performances, which can perform delicate force control. The muscle intensity of erector spinae and gluteus maximus was reduced by $40.7 \%$ and $41.1 \%$ in stooping posture and $33.0 \%$ and $41.6 \%$ in semisquatting posture. The lower back exoskeleton also uses SEA. However, this device uses two SEA units, which can provide greater force/torque compared with H-WEXv2. Then, it takes advantage of SEA's mechanical flexibility, so that its clutch can automatically release the torque between the exoskeleton and the user. MRLift utilizes a smart fluid in combination with a compression spring to produce a unique energy store-andrelease functionality. It reduces $40 \%$ of the activities of LES muscles. Muscle Suit (weight: $8 \mathrm{~kg}$ ) uses artificial muscles as actuators to help users lift and maintain a maximum load of $35 \mathrm{~kg}$. ATOUN MODEL Y (weight: $4.5 \mathrm{~kg}$ ) uses two electric actuators providing the maximum assistive force of $25 \mathrm{~N}$. Robo-Mate (weight: $11.604 \mathrm{~kg}$ ) uses elastic actuators to provide lumbar support, and its structure allows it to have a larger range of motion. Therefore, the basic carrying activities of the wearer are not restricted. SIAT-WEXv1 uses two brushless motors with harmonic drive gears to provide power and a clutch to reduce unnecessary power consumption. Active exoskeleton can provide greater assistance, but compared with passive exoskeleton, its weight is larger as shown in Figure 1.

Achieving the goal of lighter weight and greater assistance, we designed a lightweight active lumbar exoskeleton. It reduces the lumbar spine muscle activity during handling tasks, thereby reducing the risk of lumbar musculoskeletal disease (MSD). Compared with SIAT-WEXv1 [20], we designed an ergonomic lumbar support backrest that can transfer the load from the back to the hip joint. In addition, we used a quasi-direct drive to control the motion of each hip joint for assisting the hip joint to bend and extend. To increase the flexibility of the exoskeleton, the sensor information is obtained to recognize human movement and perform corresponding assistive tasks. SIAT-WEXv2 tracks the movement of the wearer smoothly; we designed a robust fuzzy adaptive control algorithm. In order to be portable, the exoskeleton weighs only $4.9 \mathrm{~kg}$.

The remainder of this paper is structured as follows: Section 2 explains the biomechanical analysis of human lifting tasks. Section 3 introduces the system design of SIATWEXv2. Subsequently, experimental procedures are provided in Section 4. The results and discussion are presented in Section 5. Lastly, in Section 6, we give a concise conclusion.

\section{Biomechanical Analysis}

Lifting actions include bending, squatting, grasping, rising, and walking. The design of ALE needs to consider the factors of the lifting motion. By analysing the kinematics and static characteristics of lifting, it can provide a theoretical basis for the design of the structure and control system. Because the mechanism of the lifting motion is very complex, researchers usually simplify the lifting model into a kinematic model and a dynamic model, as shown as Figure 2.

2.1. Kinematics Analysis of Lifting. For studying the force distribution of the lumbar spine, we established and analysed the kinematics model of lifting. Since the deformation of the lumbar spine is very small during movement, it is assumed that the lumbar spine is a nondeformable rigid body.

The lifting task can be divided into two stages: bending over and getting up. From the sagittal plane, when a person bends downward, the hip joint of the lumbar rotates clockwise, causing the lumbar spine to bend toward the pelvis; when standing, the hip joint of the lumbar rotates counter clockwise, causing the bones and lumbar spine to extend in the opposite direction of the pelvis. Therefore, the hip joint is set as the centre of motion for bending and getting up, and it is set as the centre of relative motion for the upper and lower limbs. In order to find the relationship between force and lifting process, a kinematics model was established on the sagittal plane, as shown in Figure 2(a).

Mechanical modelling of lumbar vertebrae joints, because the five-segment lumbar vertebrae joints are composed of a single structure, is extremely complicated and even impossible to solve. The motion of the lumbar joints is a joint motion, which can be simplified and approximated to a linear structure. Since the lumbar vertebrae can be flexed and extended, it is necessary to refine the analysis of the linear structure. By analysing the mechanical model of lifting, it is known that the L5-S1 joint which connects the lumbar vertebra L5 and the sacrum S1 is the farthest from the centre of gravity of the object. During users bending or lifting, the distance between the lumbar spine and the object is getting larger and larger, and the torque on the lumbar 


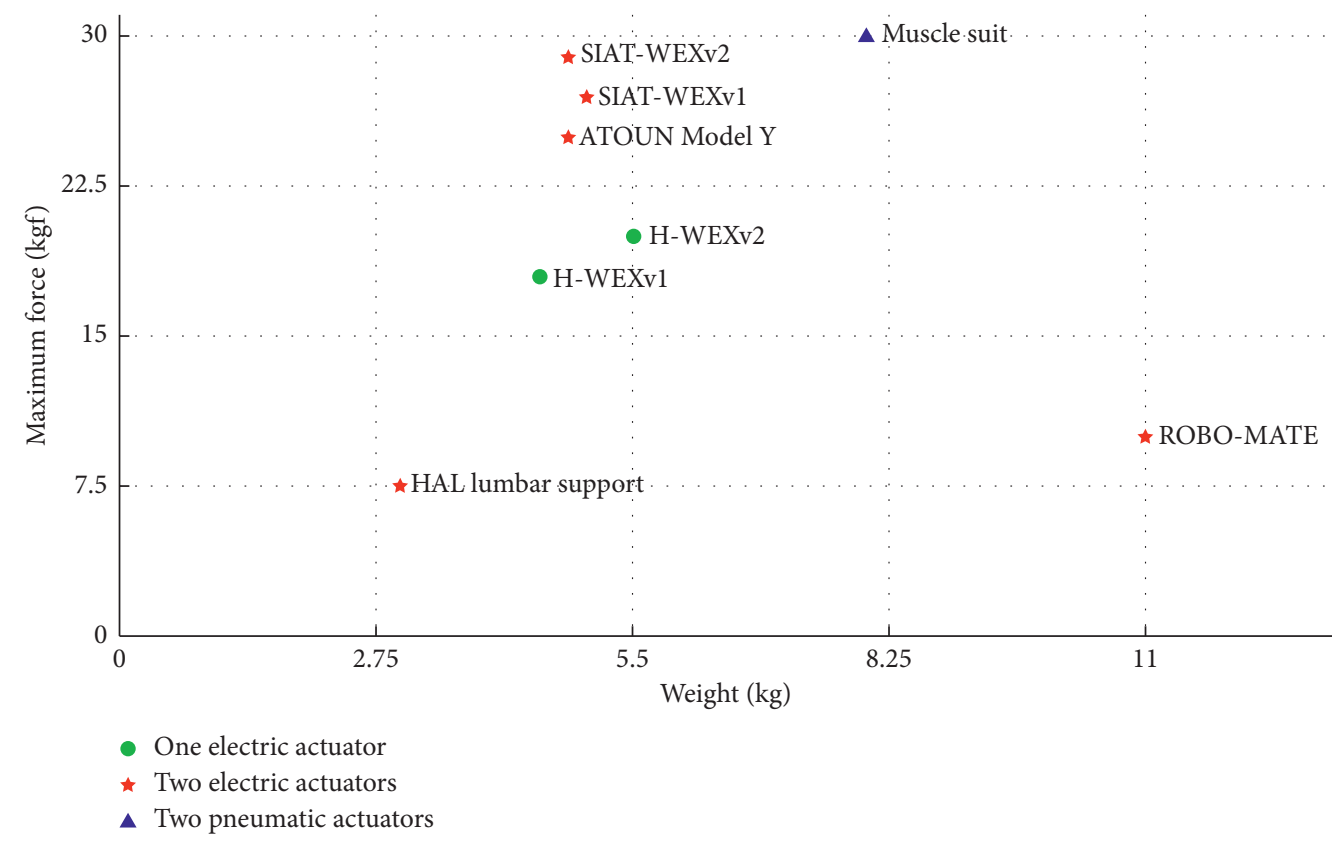

FIgURE 1: Comparison chart for maximum assistive forces with respect to weights of several lumbar exoskeletons.

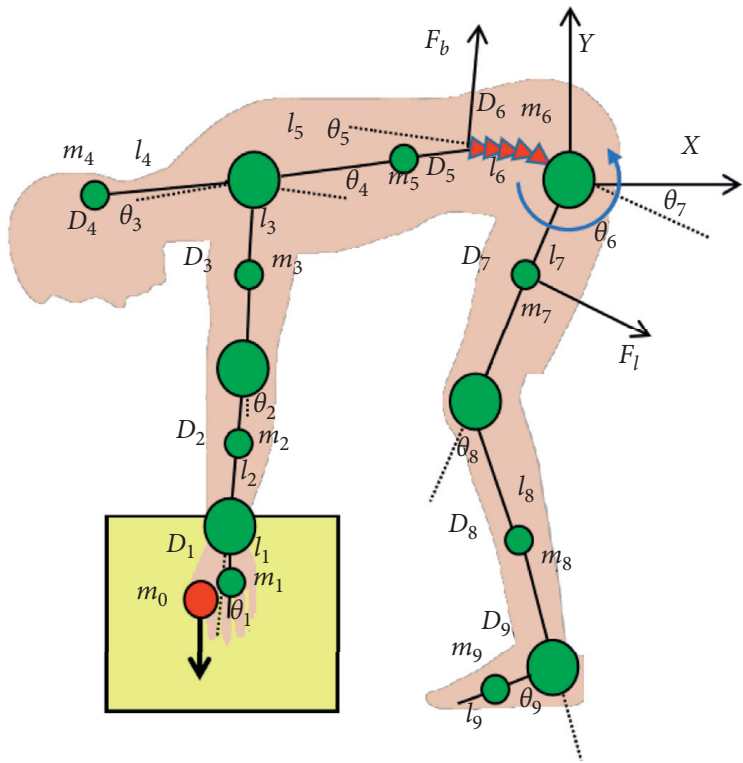

(a)

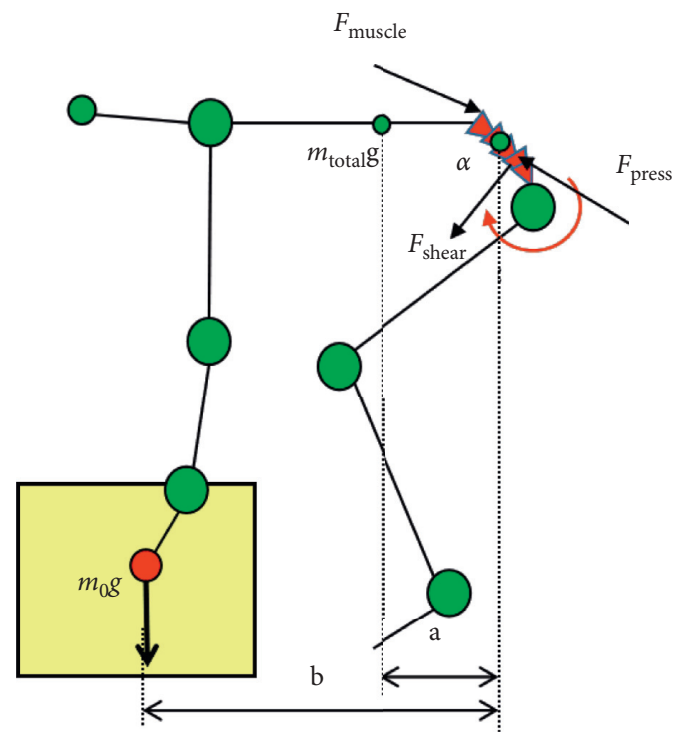

(b)

Figure 2: (a) Kinematics modelling of lifting. The green circles represent joints, all the red represent the centre of gravity of the limb, the five red triangles represent the $L_{1}-L_{5}$ lumbar vertebrae of the users from left to right, $D_{i}$ represents the centre of mass of the limb, $m_{i}$ represents the mass of limb, $l_{i}$ represents the length of the limb, and $\theta_{i}$ indicates an angle between limbs; (b) simplified mechanical model of lifting. Since the model (a) is too complicated, we simplify it to analyse its dynamic properties.

spine is increasing. So, the L5-S1 joint receives the largest moment. When the joint moment or muscle tension exceeds the limit that the L5-S1 joint can bear, the lumbar will be greatly damaged by the gravity of the object.
2.2. Mechanical Analysis of Lifting. In Figure 4, the overall mechanical structure of SIAT-WEXv2 consists of back bandages, a lumbar support backrest, hip joints, leg connections, and adjustable mechanisms 
It is assumed that the force of the mechanical model is symmetrical in the sagittal plane. The analysis shows that, at a certain moment, the various bones and joints of the users' limbs maintain static equilibrium. It can be solved by the force balance equation and moment balance equation. The following analysis is based on the biomechanical model of lifting, as shown in Figure 2(b).

The gravity of box is

$$
G_{1}=m_{0} \times g
$$

where $m_{0}$ is the mass of the box and $g$ is the gravity.

The gravity of upper body torso is

$$
G_{2}=m_{\text {total }} \times g,
$$

where $m_{\text {total }}$ is the mass of upper body torso.

Instant moments generated by weights and upper body are as follows:

$$
M_{\text {Instant }}=G_{1} \times b+G_{2} \times a,
$$

where $a$ is the horizontal distance from the centre of mass of the upper limb to the L5-S1 joint and $b$ is the horizontal distance from the centre of the box to the lumbar joint L5-S1.

Muscle torque of the users' back is

$$
M_{\text {muscle }}=F_{\text {muscle }} \times c,
$$

where $c$ is the distance from the lumbar muscle to the lumbar joint L5-S1.

The analysis result shows that the moment of the lumbar joint L5-S1 is $0 \mathrm{~N} \cdot \mathrm{m}$ :

$$
\sum M=M_{\text {muscle }}+M_{\text {Instant }} .
$$

Muscle tension can be calculated:

$$
F_{\text {muscle }}=\frac{M_{\text {muscle }}}{c}=\frac{M_{\text {Instant }}}{c}=\frac{\left(G_{1} \times b+G_{2} \times a\right)}{c},
$$

Pressure force of L5-S1 joint can also be calculated:

$$
F_{\text {press }}=G_{1} \cos \alpha+G_{2} \cos \alpha+F_{\text {muscle }} .
$$

Analysing the mechanics model of lifting and the basic dimensions of the users' limbs, it can be seen that the length of $d$ is greater than $c$, so the load pressure on the L5-S1 joint will be much greater than the total gravity of the upper body and the object. As a result, it damages the lumbar joints easily.

\section{Exoskeleton System Design}

3.1. Design Theory Analysis. When the ALE is working, the driving elements provide power to pull up the users' torso through the connection structure of the back and the legs, so that the users' legs and upper limb are kept upright. In the process of getting up, the ALE transmits the pressure on the shoulders to the legs, as shown as Figure 3(a). Therefore, comparing without ALE, the stress on the lumbar is reduced. Moreover, from the Figure 3(b), we use the dynamic model of lifting to analyse the theory of ALE [19].

The force generated by the muscles of spinal can be calculated without ALE:

$$
F_{M} d_{M}=g W_{T} d_{T} \sin (\theta)+g W_{T}\left[d_{T} \sin (\theta)+d_{L} \sin \left(\theta+\frac{\pi}{2}\right)\right]
$$

$$
R_{C}=F_{M}+g\left(W_{T}+W_{L}\right) \cos (\theta) .
$$

ALE provides power to assist with handling tasks. From Figure 3(b), the following formula is the force/torque balance formula when adding the assistive force:

$$
\begin{aligned}
& F_{M}^{\text {Pull }}=F_{M}-F_{\text {Pull }} \frac{2 \times d_{T}}{d_{M}}+g W_{X} \frac{d_{T}}{d_{M}} \sin (\theta), \\
& R_{C}^{\text {Pull }}=F_{M}^{\text {Pull }}+0+g\left(W_{T}+W_{L}+W_{X}\right) \cos (\theta) .
\end{aligned}
$$

The model paraments are defined, and the mass of the object is $20 \mathrm{~kg}$, from Section 2 [19]. Comparing the results by computing equations (8)-(11), the force provided by the muscles of the lumbar spine when wearing an exoskeleton is half of that of wearing nothing.

3.2. Overall Mechanical Structure. In Figure 4, the overall mechanical structure of SIAT-WEXv2 consists of back bandages, a lumbar support backrest, hip joints, leg connections, and adjustable mechanisms.

The back strap can ensure close contact between the lumbar support module and the users' limb and minimize the drift or movement of suit components. The lumbar support backrest includes lumbar support, hip frame, adjustment plate, hip pad, lumbar pad, and other structures. Among them, the lumbar support and the hip frame are designed as hollow titanium alloy tubes, which can ensure the strength of the lumbar support while reducing weight. The hip joint includes binding parts, flanges, brushless torque motors, and other structures. The brushless torque motor (model) is coaxially connected with the flange through the motor output shaft. The motor housing is fixed on the motor bracket, and the upper slot of the motor fixing seat is used for connecting the hip frame. The left side extension of the hollow shaft is fixedly connected with the flange, the lower groove of the flange is used for fixing the connecting plate, and the connecting plate can be connected with the leg hinge plate. The leg connection adopts a hinge structure. The two ends of the hinge are, respectively, a hinge plate and a leg connecting plate. The middle is realized by the hinge shaft to rotate freely. The hinge plate is fixed on the connecting plate. The leg connecting plate is designed with a strap buckle. The two ends of the binding buckle are, respectively, connected with two binding belts, and the binding belt is connected by the buckle, which can be quickly put on or removed. Footpads are provided where the straps are in contact with the users' thighs. The leg pads increase the 


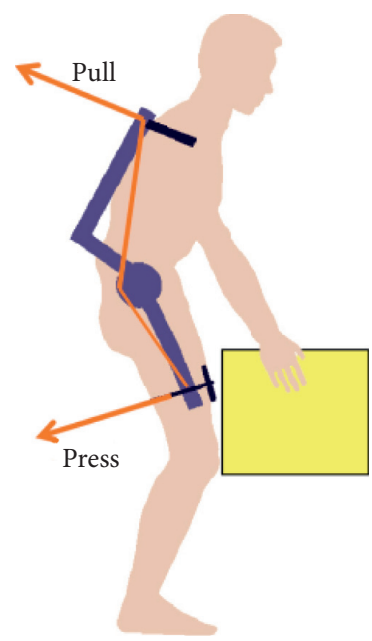

(a)

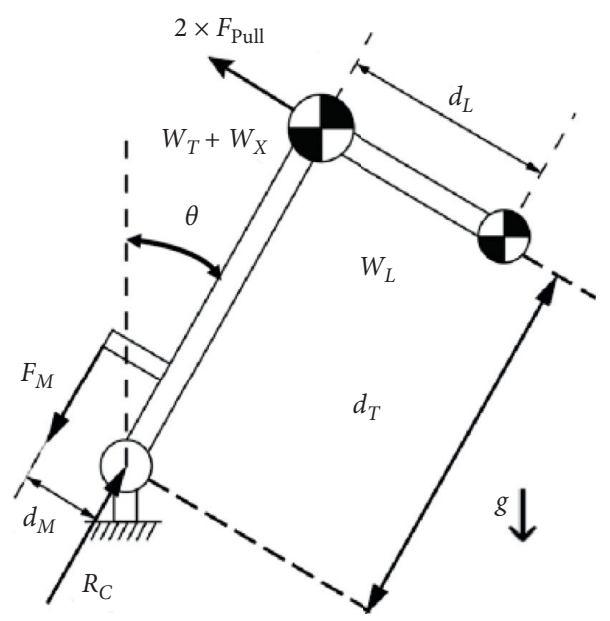

(b)

Figure 3: (a) Schematic diagram of the principle of exoskeleton assistance. (b) Mechanical model of exoskeleton assistance. The orientation angle of the torso with respect to the direction of gravity, defined as zero corresponding to upright positions and positive for forward bending; $\theta$ is the angle of users' lumbar motion; $\mathrm{F}_{\mathrm{M}}$ is the contraction force developed by the spinal muscles; $R_{C}$ is the compression on the lumbar disc, represented by joint reaction force along the torso; $W_{T}$ and $W_{L}$ are the masses of the torso and the external load, respectively; $W_{X}$ is the mass of ALE; $d_{M}$ is the distance between the rotation joint and the line of action of the muscles; $d_{T}$ is the distance of the centre of mass of the torso from the rotational joint; $d_{L}$ is the constant distance of the centre of mass of the load from that of the torso; $F_{\text {pull }}$ represents assistive force.

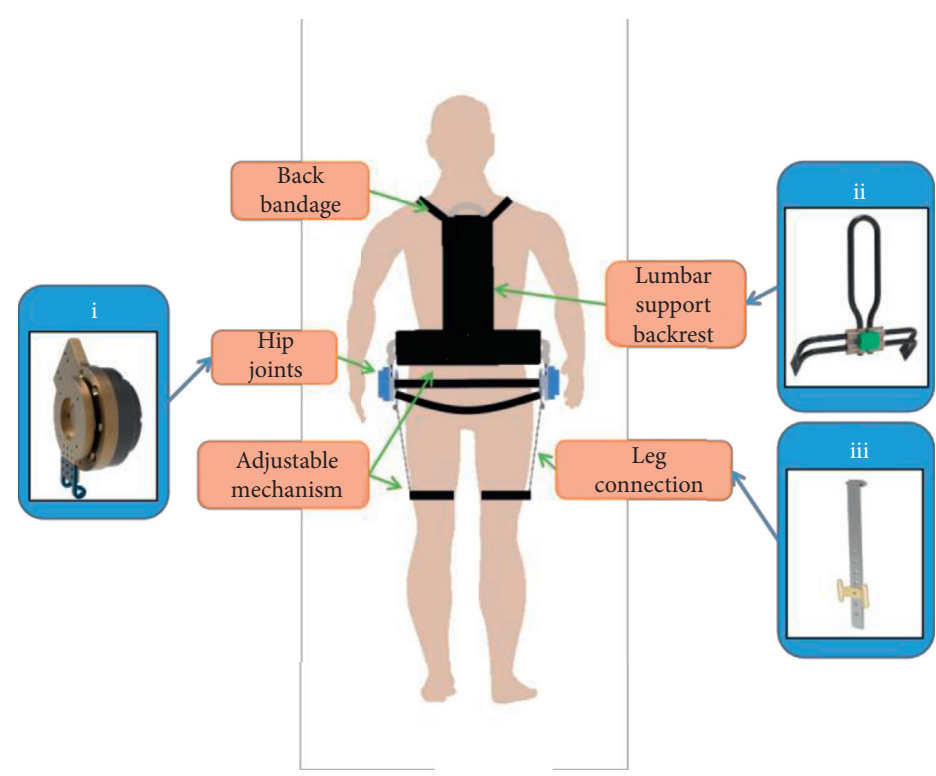

(a)

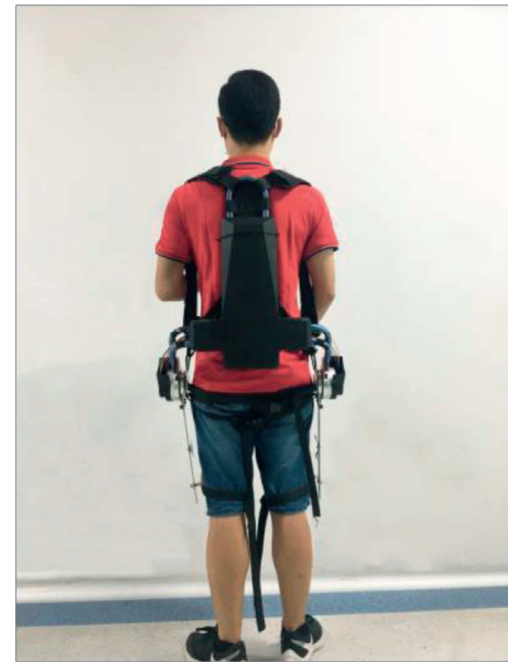

(b)

Figure 4: (a) The overall mechanical structure of SIAT-WEXv2: (i) structure model of hip joints; (ii) structure model of lumbar support backrest; (iii) structure model of leg connections. (b) Prototype of SIAT-WEXv2.

contact area between the exoskeleton and the leg skin and can effectively reduce the compressive stress.

Improving the comfort of wearing SIAT-WEXv2, the straps on the chest and legs can be adjusted through the buckle. In addition, there are adjustment holes on the leg connecting plate, so the wearer can find the best comfortable position by adjusting the straps and leg pads as he needs.
3.3. Hip Joint Module. The SIAT-WEXv2 has two hip joints. Each hip joint includes a flange, connector, and quasi-direct drive motor as shown in Figure 5(a).

The motor flange is connected to the leg connecting plate, which is a part with high-strength requirements in the exoskeleton structure. It needs to be analysed. The connection between the flange and the leg is selected as a fixed 


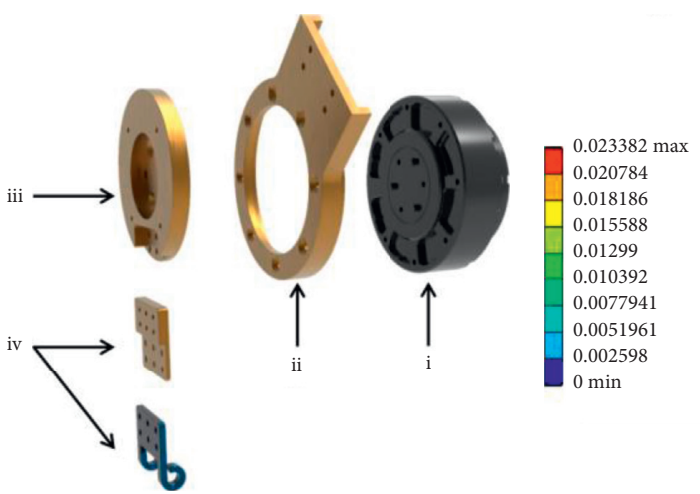

(a)

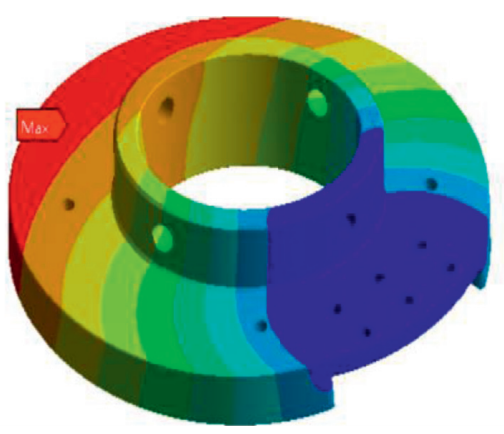

(b)

FIGURE 5: (a) Mechanical design of hip joint: (i) quasi-direct drive motor, (ii) connecting element from lumbar support backrest to hip joint, (iii) motor flange, and (iv) connecting element from hip to thigh. (b) The FEA result of total deformation of flat.

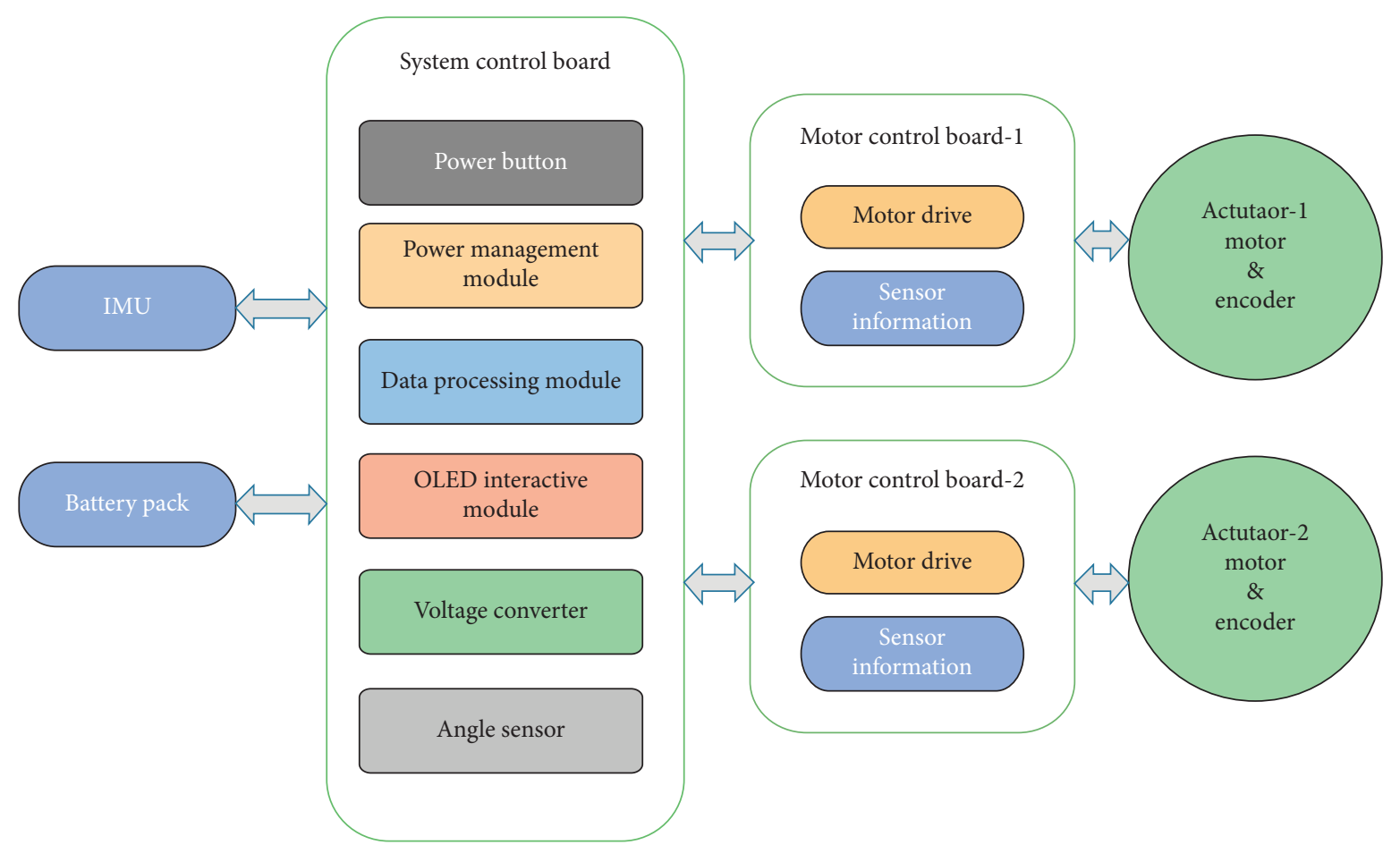

FIgURE 6: The embedded electronics system of SIAT-WEXv2. IMU, system control board, and motor control boards are installed in the lumbar support backrest; IMU and encoders are used to record the angle information of lumbar and legs, respectively; battery pack, voltage converter, and power management module can provide stable energy for the system.

constraint surface, and a torque of $60 \mathrm{~N} \cdot \mathrm{m}$ is applied to the centre surface of the flange's central axis. The results are shown in Figure 5(b). The maximum total deformation of the exoskeleton is $0.2338 \mathrm{~mm}$.

3.4. Embedded Electronics. The electrical hardware system of SIAT-WEXv2 is shown as Figure 6. It consists of an actuator unit, motor driver, system control board, and expansion module. Each actuator unit has a brushless motor and a magnetic encoder (AS5047P, repeating 16384 pulses). The motor driver (odrive 3.4) can record the angle, angular velocity, and current information of the actuator in real-time. The OLED module is used for humancomputer interaction and integrated into the exoskeleton controller. The expansion module includes IMU and battery pack.

3.5. Dynamic Model Analysis. Obtaining the exoskeleton dynamic system characteristics and then accurately controlling the motion of exoskeleton, the human-exoskeleton coupling dynamics model is established by the Euler-Lagrange method [21] as shown in Figure 7: 


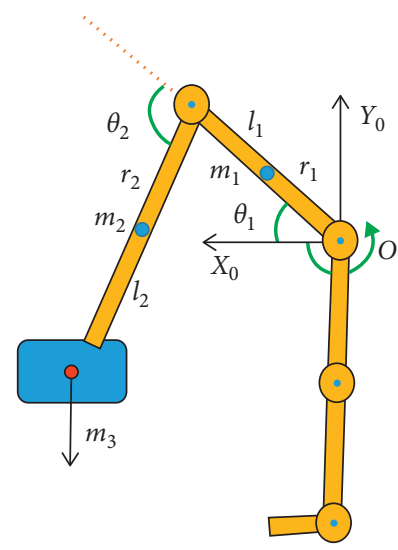

FIGURE 7: The users' upper limbs, arms, and hands are simplified. With reference to Figure 2, point $\mathrm{O}$ is selected as the origin to establish an $x_{\mathrm{o}}-y_{\mathrm{o}}$ Cartesian coordinate system; $m_{1}$ is the mass of the upper limbs and exoskeleton; $l_{1}$ is the length of the exoskeleton; $m_{2}$ is the mass of the arm; $l_{2}$ is the length of the arm; $m_{3}$ is the mass of the box.

$$
\frac{d}{d t}\left(\frac{\partial E}{\partial \dot{\theta}_{l}}\right)-\frac{\partial E}{\partial \theta_{l}}+\frac{\partial V}{\partial \theta_{l}}=\tau_{i}
$$

The relative position from link $i-1$ to link $i$ is represented by transformation matrix:

$$
{ }_{i}^{i-1} T=\left[\begin{array}{cccc}
c \theta_{i} & -s \theta_{i} & 0 & \alpha_{i-1} \\
s \theta_{i} c \alpha_{i-1} & c \theta_{i} c \alpha_{i-1} & -s \alpha_{i-1} & -s \alpha_{i-1} d_{i} \\
s \theta_{i} s \alpha_{i-1} & c \theta_{i} c \alpha_{i-1} & c \alpha_{i-1} & c \alpha_{i-1} d_{i} \\
0 & 0 & 0 & 1
\end{array}\right],
$$

And, the transformation matrix of relative position from link $i$ to link 0 is

$$
{ }_{N}^{0} T={ }_{1}^{0} T{ }_{2}^{1} T{ }_{3}^{2} T \ldots{ }_{N}^{N-1} T .
$$

The centroid position vectors of exoskeleton and users' torso and users' upper limb and object are

$$
\begin{aligned}
& { }_{1}^{0} \vec{P}=\left[\begin{array}{c}
r_{1} c_{1} \\
r_{1} s_{1} \\
0
\end{array}\right], \\
& { }_{2}^{0} \vec{P}=\left[\begin{array}{c}
l_{1} c_{1}+r_{2}\left(c_{1}-c_{2}\right) \\
l_{1} s_{1}-r_{2}\left(s_{1}-s_{2}\right) \\
0
\end{array}\right], \\
& { }_{3}^{0} \vec{P}=\left[\begin{array}{c}
l_{1} c_{1}+l_{2}\left(c_{1}-c_{2}\right) \\
l_{1} s_{1}-l_{2}\left(s_{1}-s_{2}\right) \\
0
\end{array}\right] .
\end{aligned}
$$

The angle vectors of exoskeleton and users' torso and users' upper limb and object are

$$
\begin{aligned}
& { }_{1}^{0} \vec{\theta}=\left[\begin{array}{l}
0 \\
0 \\
\theta_{1}
\end{array}\right], \\
& { }_{2}^{0} \vec{\theta}=\left[\begin{array}{c}
0 \\
0 \\
\theta_{1}+\theta_{2}
\end{array}\right], \\
& { }_{3}^{0} \vec{\theta}=\left[\begin{array}{c}
0 \\
0 \\
\theta_{1}+\theta_{2}
\end{array}\right] .
\end{aligned}
$$

The Jacobian matrixes of exoskeleton and users' torso and users' upper limb and object are

$$
{ }^{0} J_{V_{1}}=\left[\frac{\partial P_{1}}{\partial \theta_{1}} \frac{\partial P_{1}}{\partial \theta_{2}}\right]=\left[\begin{array}{cc}
-r_{1} s_{1} & 0 \\
r_{1} c_{1} & 0 \\
0 & 0
\end{array}\right],
$$$$
{ }^{0} J_{V_{2}}=\left[\frac{\partial P_{2}}{\partial \theta_{1}} \frac{\partial P_{2}}{\partial \theta_{2}}\right]=\left[\begin{array}{cc}
-l_{1} s_{1}-r_{2} s_{1} & -r_{2} s_{2} \\
l_{1} c_{1}-r_{2} c_{1} & r_{2} c_{2} \\
0 & 0
\end{array}\right],
$$$$
{ }^{0} J_{V_{3}}=\left[\frac{\partial P_{3}}{\partial \theta_{1}} \frac{\partial P_{3}}{\partial \theta_{2}}\right]=\left[\begin{array}{cc}
-l_{1} s_{1}-l_{2} s_{1} & -l_{2} s_{2} \\
l_{1} c_{1}-l_{2} c_{1} & l_{2} c_{2} \\
0 & 0
\end{array}\right] \text {, }
$$$$
{ }^{C_{1}} J_{\omega_{1}}=\left[\begin{array}{ll}
\varepsilon_{1} Z_{1} & 0
\end{array}\right]=\left[\begin{array}{ll}
0 & 0 \\
0 & 0 \\
1 & 0
\end{array}\right],
$$

$$
\begin{aligned}
& { }^{C_{2}} J_{\omega_{2}}=\left[\begin{array}{ll}
\varepsilon_{1} Z_{1} & \varepsilon_{2} Z_{2}
\end{array}\right]=\left[\begin{array}{ll}
0 & 0 \\
0 & 0 \\
1 & 1
\end{array}\right], \\
& { }^{C_{3}} J_{\omega_{3}}=\left[\begin{array}{ll}
\varepsilon_{1} Z_{1} & \varepsilon_{2} Z_{2}
\end{array}\right]=\left[\begin{array}{ll}
0 & 0 \\
0 & 0 \\
1 & 1
\end{array}\right] .
\end{aligned}
$$

If the elasticity between the components and the motion pair is not taken into account, the total kinetic energy $T$ and the potential energy $V$ of the entire mechanism are 


$$
\begin{aligned}
T= & \sum_{i} T_{i}=\frac{1}{2} \times \dot{\theta}^{T} \\
& \times\left[\sum_{i}\left(m_{i} \times{ }^{0} J_{V_{i}}^{T} \times{ }^{0} J_{V_{i}}+{ }^{C_{i}} J_{\omega_{i}}^{T} \times{ }^{C_{i}} I_{C_{i}} \times{ }^{C_{i}} J_{\omega_{i}}\right)\right] \times \dot{\theta},
\end{aligned}
$$

$V=\sum_{i=1}^{3} m_{i} g_{i}^{0} \vec{P}_{y}$

In order to facilitate calculation, the formula (18) is reduced to the following formula:

$$
T=\frac{1}{2} \times \dot{\theta}^{T} \times M \times \dot{\theta} .
$$

The mass matrix of system $M$ is

$$
M=\sum_{i}\left(m_{i} \times{ }^{0} J_{V_{i}}^{T} \times{ }^{0} J_{V_{i}}+{ }^{C_{i}} J_{\omega_{i}}^{T} \times{ }^{C_{i}} I_{C_{i}} \times{ }^{C_{i}} J_{\omega_{i}}\right) \text {. }
$$

$$
\begin{aligned}
& \frac{\mathrm{d}}{\mathrm{d} t}\left(\frac{\partial T}{\partial \dot{\theta}}\right)-\frac{\partial T}{\partial \theta}+\frac{\partial V}{\partial \theta}=\tau \\
& \frac{\partial T}{\partial \dot{\theta}}=\frac{\partial}{\partial \dot{\theta}}\left(\frac{1}{2} \times \dot{\theta}^{T} \times M \times \dot{\theta}\right)=M \dot{\theta} \\
& \frac{\mathrm{d}}{\mathrm{d} t}\left(\frac{\partial T}{\partial \dot{\theta}}\right)=M \ddot{\theta}+\dot{M} \dot{\theta} \\
& \frac{\partial T}{\partial \theta}=\frac{1}{2}\left[\begin{array}{l}
\dot{\theta}^{T} \times \frac{\partial M}{\partial \theta_{1}} \times \dot{\theta} \\
\dot{\theta}^{T} \times \frac{\partial M}{\partial \theta_{2}} \times \dot{\theta}
\end{array}\right]
\end{aligned}
$$
form:

Represent formula (22) above in/as the following matrix

Bringing formulae (19) and (20) into Lagrange's dynamic equation (22),

$$
\begin{aligned}
& {\left[\begin{array}{c}
T_{1} \\
T_{2}
\end{array}\right]=\left[\begin{array}{cc}
\left(m_{1} r_{1}^{2}+I_{1,2,3}+m_{2}\left(l_{1}^{2}+r_{2}^{2}\right)+m_{3}\left(l_{1}^{2}+l_{2}^{2}\right)+2\left(m_{2} l_{1} r_{2}+m_{3} l_{1} l_{2} c_{1}\right)\right) & 0 \\
-\left(m_{2} r_{2}^{2}-I_{2}+m_{3} l_{2}^{2}-I_{3}+\left(m_{2} l_{1} r_{2}+m_{3} l_{1} l_{2}\right) c_{1}\right) & 0
\end{array}\right]\left[\begin{array}{l}
\ddot{\theta}_{1} \\
\ddot{\theta}_{2}
\end{array}\right]} \\
& +\left[\begin{array}{cc}
0 & -\left(m_{2} r_{2}^{2}-I_{2}+m_{3} l_{2}^{2}-I_{3}+m_{2} l_{1} r_{2}+m_{3} l_{1} l_{2} c_{1}\right) \\
0 & m_{2} r_{2}^{2}+I_{2}+m_{3} l_{2}^{2}+I_{3}
\end{array}\right]\left[\begin{array}{l}
\ddot{\theta}_{1} \\
\ddot{\theta}_{2}
\end{array}\right]
\end{aligned}
$$

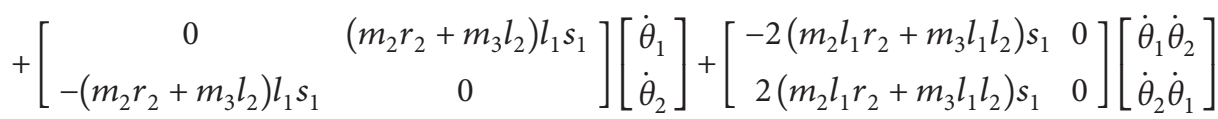

$$
\begin{aligned}
& +\left[\begin{array}{c}
\left(m_{1} r_{1}+m_{2} l_{1}+m_{3} l_{1}\right) g c_{1}+\left(m_{2} r_{2}+m_{3} l_{2}\right) g c\left(\theta_{1}-\theta_{2}\right) \\
\left(m_{2} g r_{2}+m_{3} g l_{1}\right) c\left(\theta_{1}-\theta_{2}\right)
\end{array}\right] \text {. }
\end{aligned}
$$

During the process of lifting heavy objects, the objects are always vertically downwards and the geometry is $\theta_{2}=\theta_{1}+(\pi / 2)$.

Substituting into the above formula (26), we get

$$
\begin{aligned}
{[T]=} & {\left[m_{1} r_{1}^{2}+I_{1}+m_{2} l_{1}^{2}+m_{3} l_{1}^{2}+4\left(I_{2}+I_{3}\right)\right]\left[\ddot{\theta}_{1}\right] } \\
& +\left[\left(m_{1} g r_{1}+m_{2} g l_{1}+m_{3} g l_{1}\right) \cos \theta_{1}\right] .
\end{aligned}
$$

3.6. Control Strategy. To improve the versatility of exoskeleton system, the control strategy could be considered. Without considering friction and other disturbances, the dynamic equation of ALE can be described by

$$
H(q) \ddot{q}+C(q, \dot{q}) \dot{q}+G(q)=T,
$$

where $q$ is the joint angle, $H(q)$ is the inertia matrix of the human-exoskeleton system, $C(q, \dot{q})$ is the centripetal force and Coriolis force, $G(q)$ is the moment formed by gravity, $T$ is the torque or force applied to each joint, and $q_{d}$ is the desired angle of the intended movement.

The weight uncertainty of the object and the wearer affects the stability of the exoskeleton system. A new control method uses a fuzzy adaptive algorithm to reduce the influence of the above two factors on the system. Then, a fuzzy compensator is used to approximate these two interference terms. Based on this, fuzzy adaptive control through modelbased control algorithms can be defined as [22]

$$
\begin{gathered}
D(q) \ddot{q}+C(q, \dot{q}) \dot{q}+G(q)+e(q, \ddot{q}, t)=\tau, \\
D(q)=D\left(m_{a}, q\right), C(q, \dot{q})=C\left(m_{a}, q, \dot{q}\right), G(q)=G\left(m_{a}, q\right),
\end{gathered}
$$

$$
\begin{aligned}
e(q, \ddot{q}, t) & =e_{D}[D(q) \ddot{q}]+e_{G}[G(q)] \\
& =D\left(m_{a}, q\right) \ddot{q}-D\left(m_{d}, q\right) \ddot{q}+G\left(m_{a}, q\right)-G\left(m_{d}, q\right)
\end{aligned}
$$


where $m_{d}$ is the nominal value which is known, $m_{a}$ is the actual value of the model, $e_{D}$ is the error of inertial force, and $e_{G}$ is the error of model gravity:

$$
F(q, \ddot{q})=e_{D}[D(q) \ddot{q}]+e_{G}[G(q)] .
$$

The uncertain elements of system of lumbar exoskeleton include the variation of workload and the mass of the system. Considering those reasons, the function $\mathrm{F}$ is used to represent the uncertain part of the system. The fuzzy adaptive control equation is determined and is defined as

$$
\tau=D(q) \ddot{q}+C(q, \dot{q}) \dot{q}+G(q)+\widehat{F}(q, \ddot{q} \mid \Theta)-K_{D} s .
$$

The adaptive law is defined as

$$
\dot{\Theta}=-\Gamma^{-1} s \xi(q, \ddot{q}) \text {. }
$$

Using the above definition, we can introduce the Lyapunov function:

$$
V(t)=\frac{1}{2}\left(s^{T} D s+\widetilde{\Theta}^{T} \Gamma \widetilde{\Theta}\right) .
$$

Then, we compute the time derivative of $V$ and substitute equation (35) into, we can obtain

$$
\dot{V}(t)=-s^{T}\left(D \ddot{q}_{d}+C \dot{q}_{d}+G\right)+\widetilde{\Theta}^{T} \Gamma \dot{\widetilde{\Theta}} .
$$

For simplifying the function of time derivative of $V$, fuzzy approximation error is defined as

$$
w=F(q, \ddot{q})-\widehat{F}(q, \ddot{q} \mid \Theta) \text {. }
$$

Equation (36) is substituted into equation (35), and the final function of time derivative of $V$ is

$$
\begin{aligned}
\dot{V}(t) & =-s^{T} K_{D} s-s^{T} w+\widetilde{\Theta}^{T} \Gamma \dot{\widetilde{\Theta}}-s \widetilde{\Theta}^{T} \xi^{2}(q, \ddot{q}) \\
& =-s^{T} K_{D} s-s^{T} w .
\end{aligned}
$$

According to LaSalle invariance principle, $s \longrightarrow 0$ when $\dot{V} \equiv 0, s \equiv 0$, and $t \longrightarrow \infty$. Then, the convergence rate of the system depends on $K_{\mathrm{D}}$. Due to $V \geq 0, \dot{V} \geq 0$, and $V$ bounded, $\widetilde{\Theta}$ bounded, but $\widetilde{\Theta}$ is not necessarily converged to 0 . In that case, eliminating approximation errors, a robust adaptive law is designed as

$$
\begin{aligned}
\tau= & D(q) \ddot{q}+C(q, \dot{q}) \dot{q}+G(q)+\widehat{F}(q, \ddot{q} \mid \Theta)-K_{D} s \\
& -W \operatorname{sgn}(s),
\end{aligned}
$$

where $W$ is obtained as

$$
W=\operatorname{diag}[w], w \gg|w|+\left|w^{2}\right| .
$$

The global stability proof of the algorithm has a detailed derivation process in the literature [23]. The block diagram of the control is shown in Figure 8.

\section{Experiment}

4.1. Participants. Ten subjects participated in this study, and their physical information is listed in Table 1. Before the trial, these volunteers read the experimental protocol and signed a consent form. The test was conducted in accordance with the Research Ethics Procedures of the Shenzhen Institute of Advanced Technology.

4.2. Testing Equipment and Surface Electromyography. The Surface EMG Sensor (SX230) of Biometrics Ltd (Sampling rate: $500 \mathrm{~Hz}$ ) is an active sensor with the amplifier's input impedance over 10,000,00 M Ohms, pairing it with the data acquisition product the DataLOG (MWX8). SX230 can obtain and display surface EMG data accurately, as shown Figure 9(a). In our experiment, we only measure the LES at the level of L3 vertebrae with an interval of $4 \mathrm{~cm}$; the position of EMG sensors is shown in Figure 9(b); the reference electrode was placed on the elbow joint, as shown Figure 9(c). Before the EMG electrodes were fixed at the above positions, the skin was cleaned with an alcohol cotton ball. After electrodes have attached to the skin, we wrapped a circle of the medical belt for improving the accuracy of EMG data.

4.3. Testing Procedures. Before starting each test, we adjusted the back bandage, thigh bandage, and adjustable mechanism. Second, we verified the DataLOG connection. Then, the subject was asked to lift the box. As the experiment progresses, the load will be gradually increased from $0 \mathrm{~kg}$ to $25 \mathrm{~kg}$ by $5 \mathrm{~kg}$ at equal weight intervals. Each volunteer wearing or not wearing an exoskeleton lifted 6 levels of weights per round, and there was a 15 -minute pause between two weightlifting tasks to ensure that the volunteers' LES muscles were fully rested. Figure 10 shows the test process.

\section{Results and Discussion}

After filtering the EMG signal with a band-pass filter (bandwidth of $20-460 \mathrm{~Hz}$ ) and a band-stop filter (eliminating power frequency noise), we calculated the integrated EMG (iEMG) value during lifting and we defined the assistance efficiency of the exoskeleton using the following formula:

$$
E=1-\frac{I_{E}}{I}
$$

where $I_{E}$ and $I$ are iEMG when the subjects lift the loads with/without SIAT-WEXv2.

According to the initial statistical analysis, there is no significant difference between the EMG signals of the left and right muscles. Therefore, the EMG data of the left and 


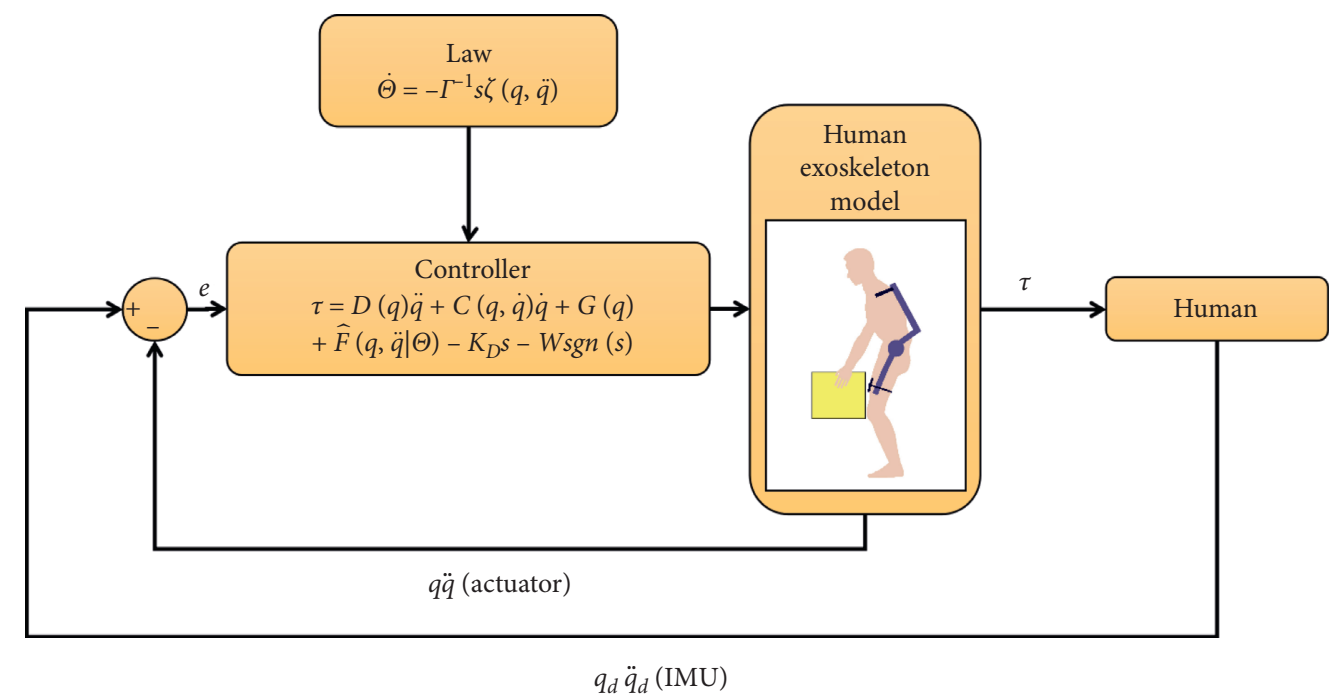

FIgURE 8: The block diagram of robust fuzzy adaptive control of human exoskeleton system.

TABLE 1: The statistical data of subject's personal information.

\begin{tabular}{lccc}
\hline & Medium & Minimum & Maximum \\
\hline Height $(\mathrm{cm})$ & 170 & 160 & 184 \\
Weight $(\mathrm{kg})$ & 67 & 60 & 80 \\
Age (years) & 30 & 22 & 45 \\
\hline
\end{tabular}

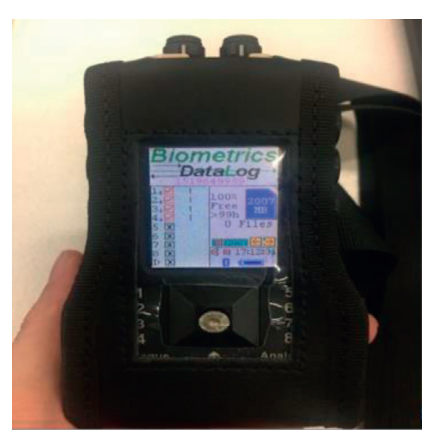

(a)

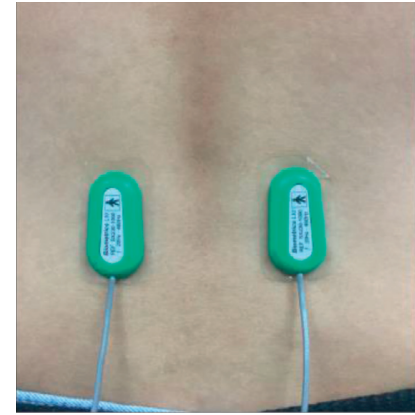

(b)

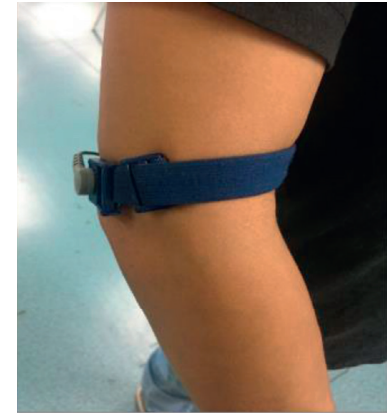

(c)

FIGURE 9: Testing equipment and testing lumbar position. (a) The surface EMG sensor (SX230) of Biometrics Ltd; (b) the position of EMG sensors; (c) the reference electrode position.

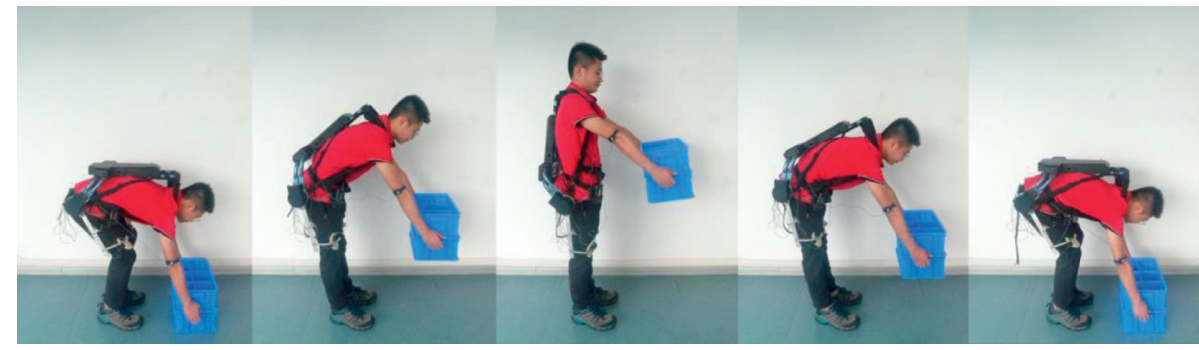

FIgURE 10: The process of a wearer lifting. 


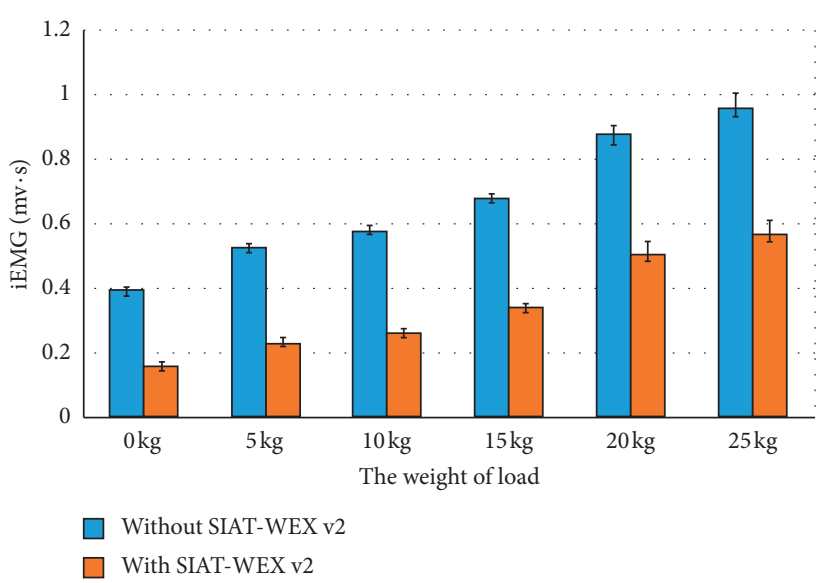

(a)

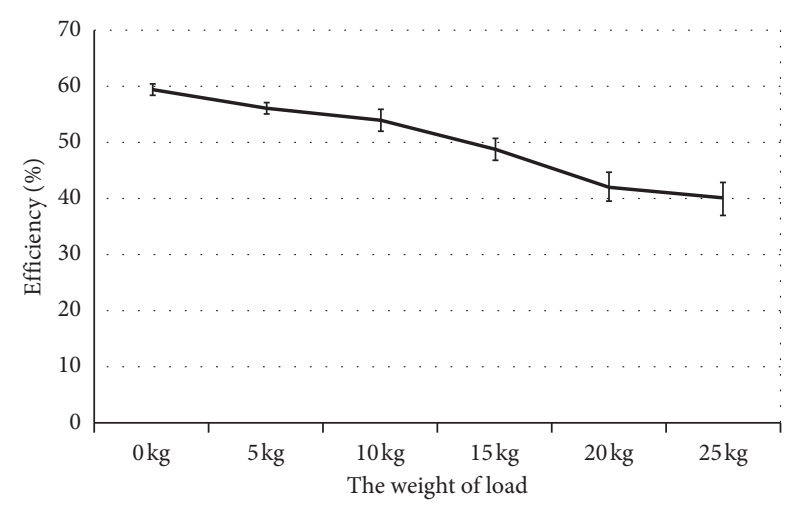

(b)

FIgUre 11: (a) iEMG of LES with loads from $0 \mathrm{~kg}$ to $25 \mathrm{~kg}$; (b) efficiency with SIAT-WEXv2.

TABLE 2: Comparison of the reduction of the three kinds of exoskeleton myoelectric activity.

\begin{tabular}{lc}
\hline Exoskeleton & Lumbar erector spinae (\%) \\
\hline APO [24] & 30 \\
H-WEXv2 [14] & $40.7-41.1$ \\
SIAT-WEXv2 & 48 \\
\hline
\end{tabular}

right muscles will be merged, and then iEMG will be taken. From Figure 11(a), the difference of iEMG during lifting with/without SIAT-WEXv2 is obviously seen. Figure 11(b) shows the reduction of iEMG signal decreased monotonically from $60 \% \pm 5.5 \%$ to $40.5 \% \pm 6.5 \%$. Especially, during users lifting $25 \mathrm{~kg}$ loads, the reduction rate of iEMG signal can maintain $40.5 \% \pm 6.5 \%$. Thereby, SIAT-WEXv2 can reduce the incidence of low back pain and lumbar muscle strain caused by repeated and longterm weightlifting.

It can be seen from the Table 2, when the user wears SIAT-WEXv2 to carry $15 \mathrm{~kg}$ objects, their lumbar muscle activity is reduced the most among three ALEs. This shows that SIAT-WEXv2 has a better assistive effect.

In the developed ALEs, SIAT-WEXv2 has a lighter mechanical structure, smooth power assistance, and greater power assistance value. First of all, we not only optimized the mechanical structure but also reduced its total weight to $4.98 \mathrm{~kg}$. In addition, it obtains real-time angle information through the IMU and motor encoder, and the robust fuzzy adaptive control algorithm allows the actuator to follow the user's motion trajectory smoothly. Finally, experimental results show that SIAT-WEXv2 can reduce the muscle fatigue of the lumbar spinae during users' lifting.

The exoskeleton proposed in this study has two limitations. First, SIAT-WEXv2 follows the trajectory of the wearer's movement to provide assistance, without fully considering the human-computer interaction. In this regard, new control strategies should be considered to improve their adaptability. Then, our experiment is limited to the laboratory. In industrial applications, material handling workers conduct experimental tests. According to the experimental results, the next step is to collect data according to different application scenarios for multiscene recognition research. Ultimately, SIAT-WEXv2 can be applied to more fields, especially in daily life.

\section{Conclusions}

This work introduced a new ALE, SIAT-WEXv2, featuring with simple structure, light weight, and prominent assistive capacity based on ergonomics design concept and biomechanical analysis method. In terms of control algorithms, we used a robust fuzzy adaptive control algorithm to make the actuator follow the wearer's movement smoothly which means the output assistive force can dynamically match the changes of lifting load and the differences of gravity centre position of wearer's upper body. We carried out rigorous tests to evaluate the performance SIAT-WEXv2 by measuring the reduction of iEMG signal of LES. When lifting different loads with weight ranging from $0-25 \mathrm{~kg}$, the reduction of iEMG signal decreased monotonically from $60 \% \pm 5.5 \%$ to $40.5 \% \pm 6.5 \%$. Therefore, SIAT-WEXv2 would be a good solution to reduce the risk of waist injuries during the heavy object lifting task.

\section{Data Availability}

The data used to support the findings of this study are included within the article.

\section{Conflicts of Interest}

There are no conflicts of interest regarding the publication of this paper.

\section{Acknowledgments}

This work was partially supported by the Key-Area Research and Development Program of Guangdong Province (2019B090917002). 


\section{References}

[1] R. Prins, P. Jensen, T. Lord et al., Inventory of Socio-Economic Information about Work-Related Musculoskeletal Disorders in the Member States of the European Union, European Agency for Safety and Health at Work, Bilbao, Spain, 2000.

[2] S. Toxiri, M. B. Näf, M. Lazzaroni et al., "Back-support exoskeletons for occupational use: an overview of technological advances and trends," IISE Transactions on Occupational Ergonomics and Human Factors, vol. 7, no. 3-4, pp. 237-249, 2019.

[3] A. S. Koopman, S. Toxiri, V. Power et al., "The effect of control strategies for an active back-support exoskeleton on spine loading and kinematics during lifting," Journal of Biomechanics, vol. 91, pp. 14-22, 2019.

[4] A.-E. Mohammad, M. J. Agnew, and J. M. Stevenson, "An onbody personal lift augmentation device (PLAD) reduces EMG amplitude of erector spinae during lifting task," Clinical Biomechanics, vol. 7, pp. 237-249, 2019.

[5] C. A. Lotz, M. J. Agnew, A. A. Godwin, and J. M. Stevenson, "The effect of an on-body personal lift assist device (PLAD) on fatigue during a repetitive lifting task," Journal of Electromyography and Kinesiology, vol. 19, no. 2, pp. 331-340, 2009.

[6] R. B. Graham, C. L. W. Smallman, E. M. Sadler, and J. M. Stevenson, "Interjoint coordination and the personal liftassist device," Journal of Applied Biomechanics, vol. 29, no. 2, pp. 194-204, 2013.

[7] W. Michael, R.. David, and K.. Homayoon, "Lower extremity exoskeleton reduces back forces in lifting," in Proceedings of the ASME 2009 Dynamic Systems and Control Conference, American Society of Mechanical Engineers Digital Collection, Hollywood, CA, USA, pp. 49-56, October 2009.

[8] B. L. Ulrey and F. A. Fathallah, "Subject-specific, whole-body models of the stooped posture with a personal weight transfer device," Journal of Electromyography and Kinesiology, vol. 23, no. 1, pp. 206-215, 2013.

[9] M. M. Alemi, J. Geissinger, A. A. Simon, S. E. Chang, and A. T. Asbeck, "A passive exoskeleton reduces peak and mean EMG during symmetric and asymmetric lifting," Journal of Electromyography and Kinesiology, vol. 47, pp. 25-34, 2019.

[10] S. J. Baltrusch, J. H. van Dieën, A. S. Koopman, C. RodriguezGuerrero, J. Babič, and H. Houdijk, "SPEXOR passive spinal exoskeleton decreases metabolic cost during symmetric repetitive lifting," European Journal of Applied Physiology, vol. 120, no. 2, pp. 401-412, 2020.

[11] M. B. Näf, A. S. Koopman, B. Saskia, R.-G. Carlos, V. Bram, and L. Dirk, "Passive back support exoskeleton improves range of motion using flexible beams," Frontiers in Robotics and $A I$, vol. 5, p. 72, 2018.

[12] H. Kazerooni, W. Pillai, and P.. Minerva, "Evaluation of trunk-supporting exoskeleton," Proceedings of the Human Factors and Ergonomics Society Annual Meeting, vol. 63, no. 1, pp. 1080-1083, 2019.

[13] H. K. Ko, S. W. Lee, D. H. Koo, I. Hyun, and H. Dong Jin, "Waist-assistive exoskeleton powered by a singular actuation mechanism for prevention of back-injury," Robotics and Autonomous Systems, vol. 107, pp. 1-9, 2018.

[14] D. J. Hyun, H. Lim, S. Nam, and N.. Seungkyu, "Singular wiredriven series elastic actuation with force control for a waist assistive exoskeleton, H-WEXv2," IEEE/ASME Transactions on Mechatronics, vol. 25, no. 2, pp. 1026-1035, 2020.

[15] T. Zhang and H. Huang, "A lower-back robotic exoskeleton: industrial handling augmentation used to provide spinal support," IEEE Robotics \& Automation Magazine, vol. 25, no. 2, pp. 95-106, 2018.

[16] H.. Modar, K.. Maxwell, Y.. Keisuke, K.. Hideki, M.. Hiromi, and S.. Kenji, "MR lift: a semi-active lower back support exoskeleton based on MR fluid and force retention technology," in Proceedings of the 2019 IEEE/RSJ International Conference on Intelligent Robots and Systems (IROS), pp. 7349-7354, Macau, China, November 2019.

[17] A.. Takamitsu, N.. Hirokazu, and K.. Hiroshi, "Development of muscle suit and application to factory laborers," in Proceedings of the 2009 International Conference on Mechatronics and Automation, pp. 1027-1032, Changchun, China, August 2009.

[18] W.. Jacob, "Exoskeleton exploration: research, development, and applicability of industrial exoskeletons in the automotive industry," 2018.

[19] T.. Stefano, O.. Jesus, M.. Jawad, F.. Jorge, L. A. Mateos, and D. G. Caldwell, "A wearable device for reducing spinal loads during lifting tasks: biomechanics and design concepts," in Proceedings of the 2015 IEEE International Conference on Robotics and Biomimetics (ROBIO), pp. 2295-2300, Zhuhai, China, December 2015.

[20] X. Yong, Z. Yan, C. Wang, C. Wang, N. Wu, and W. Xinyu, "Ergonomic mechanical design and assessment of a waist assist exoskeleton for reducing lumbar loads during lifting task," Micromachines, vol. 10, no. 7, p. 463, 2019.

[21] J. J. Craig, Introduction to Robotics: Mechanics and Control, 3/E, Pearson Education India, Noida, India, 2009.

[22] J. J. Slotine and E. Li, Weiping and Others, Applied Nonlinear Control, Prentice Hall Englewood Cliffs, NJ, 1991.

[23] Y. Byung Kook and H. Woon Chul, "Adaptive control of robot manipulator using fuzzy compensator," IEEE Transactions on Fuzzy Systems, vol. 8, pp. 186-199, 2000.

[24] C.. Baojun, G.. Lorenzo, L.. Francesco, V.. Nicola, and C.. Simona, "A real-time lift detection strategy for a hip exoskeleton," Frontiers in Neurorobotics, vol. 12, p. 17, 2018. 